\title{
Iterative Receiver Design for MIMO Systems with Improper Signal Constellations
}

\author{
Pei Xiao, Mathini Sellathurai \\ The Institute of Electronics, Communications and Information Technology \\ Queen's University Belfast, BT3 9DT, United Kingdom \\ E-mail: \{pei.xiao,m.sellathurai\}@ecit.qub.ac.uk
}

\begin{abstract}
In this paper, we propose a novel iterative receiver strategy for uncoded multiple-input, multiple-output (MIMO) systems employing improper signal constellations. The proposed scheme is shown to achieve superior performance and faster convergence without the loss of spectrum efficiency compared to the conventional iterative receivers. The superiority of this novel approach over conventional solutions is verified by the simulation results.
\end{abstract}

\section{INTRODUCTION}

Recent studies have shown that the use of multiple antennas in a wireless communication system significantly improves the system's spectral efficiency, enables a growth in transmission rate linear in the minimum number of antennas at either end [1], [2], and improves link reliability and coverage [3]. However, the main problem for transmission over multipleinput, multiple-output (MIMO) channels is the separation or equalization of the parallel data streams. In order to exploit the capacity and performance gains promised by MIMO, we must deal with the co-antenna interference (CAI). It was shown in [2], [4] that iterative (turbo) detection provides an effective means to combat CAI and to approach the capacity offered by the MIMO systems. In its original form, the iterative receiver employs the maximum a posteriori probability (MAP) algorithm [5], [6], which has a high computational complexity that increases exponentially with the spatial diversity and modulation orders. To reduce the complexity, a MIMO turbo receiver based on soft interference cancellation was proposed in [4], [7], [8]. The basic idea is to iteratively cancel out the CAI with soft symbol estimates and suppress the residual interference with a zero-forcing (ZF) or minimummean-square-error (MMSE) filter. We call this method 'ICZF/MMSE' in the sequel. The main computational complexity of this approach is incurred by the matrix inverse in the filter coefficient computation, which is much simpler than the MAP algorithm.

In this paper, we show that the existing iterative receiver designs are sub-optimum for systems employing improper modulation schemes and their performance can be improved by exploitation of the improperness of signal constellation. The proposed schemes are compared to their conventional counterparts, and are shown to have superior performance without any loss of spectrum efficiency.

Throughout this paper, $(\cdot)^{\mathcal{T}}$ denotes matrix transpose, $(\cdot)^{\mathcal{H}}$ matrix conjugate transpose, $(\cdot)^{*}$ matrix conjugate, $\mathrm{E}[\cdot]$ expectation, $\|\cdot\|$ Euclidean norm, $\|\cdot\|_{F}$ Frobenius norm, $\operatorname{Tr}(\cdot)$ trace operation, and $\mathbf{I}_{N}$ an $N \times N$ identity matrix.

\section{SYSTEM MODEL}

We denote $N_{t}, N_{r}$ as the number of transmit and receive antennas, respectively. The transmitted symbol vector $\mathbf{s}=$ $\left[\begin{array}{lll}s_{1} & \ldots & s_{N_{t}}\end{array}\right]^{\mathcal{T}}$ comprises the transmit symbol of $N_{t}$ parallel data streams. These streams can be due to a parallel (layered) encoding of a high-rate data signal, or they may belong to different and independent users. The data symbols are assumed to be uncorrelated and to have zero mean and identical energy $\sigma_{s}^{2}$, i.e., $\mathrm{E}\left[\mathbf{s s}^{\mathcal{H}}\right]=\sigma_{s}^{2} \mathbf{I}_{N_{t}}$. The received signal can be expressed as

$$
\mathbf{r}=\mathbf{H s}+\mathbf{v}=\sum_{i=1}^{N_{t}} \mathbf{h}_{i} s_{i}+\mathbf{v} \in \mathbb{C}^{N_{r} \times 1},
$$

where $\mathbf{r}=\left[\begin{array}{llll}r_{1} & r_{2} & \ldots & r_{N_{r}}\end{array}\right]^{\mathcal{T}}$ is the received signal vector; $\mathbf{v}=\left[\begin{array}{llll}v_{1} & v_{2} & \ldots & v_{N_{r}}\end{array}\right]^{\mathcal{T}}$ denotes the complex additive white Gaussian noise vector with zero mean and covariance matrix $\sigma_{v}^{2} \mathbf{I}_{N_{r}}$, i.e., $\mathbf{v} \sim \mathcal{C N}\left(\mathbf{0}, \sigma_{v}^{2} \mathbf{I}_{N_{r}}\right)$. The channel matrix $\mathbf{H} \in \mathbb{C}^{N_{r} \times N_{t}}$ contains the complex channel gains between every transmit and receive antenna pair, and $\mathbf{h}_{i}$ is the $i$ th column of $\mathbf{H}$. We assume uncorrelated Rayleigh fading channel model, and the channel coefficients are independent complex Gaussian random variables.

Suppose the symbol $s_{n}$ is to be decoded. According to (1), the received vector after interference cancellation is given as

$$
\mathbf{r}_{n}=\mathbf{r}-\mathbf{H} \overline{\mathbf{s}}_{n}=\mathbf{H}\left[\mathbf{s}-\overline{\mathbf{s}}_{n}\right]+\mathbf{v} \in \mathbb{C}^{N_{r} \times 1},
$$

where $\mathbf{r}_{n}$ is the interference canceled version of $\mathbf{r}$, and

$$
\begin{aligned}
\mathbf{s} & =\left[\begin{array}{lllllll}
s_{1} & \ldots & s_{n-1} & s_{n} & s_{n+1} & \ldots & s_{N_{t}}
\end{array}\right]^{\mathcal{T}} ; \\
\overline{\mathbf{s}}_{n} & =\left[\begin{array}{lllllll}
\bar{s}_{1} & \ldots & \bar{s}_{n-1} & 0 & \bar{s}_{n+1} & \ldots & \bar{s}_{N_{t}}
\end{array}\right]^{\mathcal{T}} .
\end{aligned}
$$

The vector $\overline{\mathbf{s}}_{n}$ contains the soft estimate of the interference symbols from the previous iteration. The derivation of $\overline{\mathbf{s}}_{n}$ will be given later on.

Note that (2) represents a decision-directed iterative scheme, where the detection procedure at the the $p^{\text {th }}$ iteration uses the symbol estimates from the $(p-1)^{t h}$ iteration. The performance is improved in an iterative manner due to the fact that the symbols are more accurately estimated (leading to better interference cancellation) as the iterative procedure goes on. For simplicity, the iteration index is omitted, whenever no ambiguity arises.

In order to further suppress the residual interference in $\mathbf{r}_{n}$, an instantaneous linear filter is applied to $\mathbf{r}_{n}$, to obtain $z_{n}=\mathbf{w}_{n}^{\mathcal{H}} \mathbf{r}_{n}$, where the filter coefficient vector $\mathbf{w}_{n} \in \mathbb{C}^{N_{r} \times 1}$ 
is chosen by minimizing $e_{n}=\mathrm{E}\left\{\left|\mathbf{w}_{n}^{\mathcal{H}} \mathbf{r}_{n}-s_{n}\right|^{2}\right\}$ or $e_{n}=$ $\mathrm{E}\left\{\left|\mathbf{w}_{n}^{\mathcal{H}}\left(\mathbf{r}_{n}-\mathbf{v}\right)-s_{n}\right|^{2}\right\}$, respectively, under the MMSE and $\mathrm{ZF}$ criteria. It can be derived as

$$
\begin{aligned}
& \mathbf{w}_{n}=\sigma_{s}^{2}\left[\mathbf{H} \mathbf{V}_{n} \mathbf{H}^{\mathcal{H}}+N_{0} \mathbf{I}\right]^{-1} \mathbf{h}_{n} \\
& \mathbf{w}_{n}^{\prime}=\sigma_{s}^{2}\left[\mathbf{H} \mathbf{V}_{n} \mathbf{H}^{\mathcal{H}}\right]^{-1} \mathbf{h}_{n}
\end{aligned}
$$

for MMSE;

for ZF.

The matrix $\mathbf{V}_{n} \in \mathbb{R}^{N_{t} \times 1}$ is formed as

$\left.\mathbf{V}_{n}=\operatorname{diag}\left\{\operatorname{var}\left(s_{1}\right) \ldots \operatorname{var}\left(s_{n-1}\right) \quad \sigma_{s}^{2} \quad \operatorname{var}\left(s_{n+1}\right) \ldots \operatorname{var}\left(s_{N_{t}}\right)\right]\right\}$,

where $\operatorname{var}\left(s_{j}\right)=\mathrm{E}\left[\left|s_{j}-\bar{s}_{j}\right|^{2}\right]$. Refer to [4], [7]-[10] for a detailed description of this conventional algorithm.

\section{IMPROVED ITERATIVE SOLUTION}

The filter design shown in (4) is optimum for systems with proper modulations, such as $M$-QAM and $M$-PSK (for which $\left.E\left[\mathbf{s s}^{\mathcal{T}}\right]=\mathbf{0}\right)$. However, for the improper modulation schemes considered in this paper, such as $M$-ary ASK, OQPSK (for which $E\left[\mathbf{s s}^{\mathcal{T}}\right] \neq \mathbf{0}$ ), the above mentioned design criterion is sub-optimum. We propose a new scheme based on an error criterion defined by

$$
\begin{aligned}
e_{n}= & \operatorname{Re}\left\{\mathbf{g}_{n}^{\mathcal{H}}\left(\mathbf{H}\left[\mathbf{s}_{n}-\overline{\mathbf{s}}_{n}\right]+\mathbf{v}\right)\right\}-s_{n} \\
= & 0.5 \mathbf{g}_{n}^{\mathcal{H}} \mathbf{H}\left[\mathbf{s}_{n}-\overline{\mathbf{s}}_{n}\right]+0.5\left(\mathbf{g}_{n}^{\mathcal{H}} \mathbf{H}\right)^{*}\left[\mathbf{s}_{n}-\overline{\mathbf{s}}_{n}\right] \\
& +0.5\left[\mathbf{g}_{n}^{\mathcal{H}} \mathbf{v}+\left(\mathbf{g}_{n}^{\mathcal{H}} \mathbf{v}\right)^{*}\right]-s_{n} .
\end{aligned}
$$

Since only the real part of this output is relevant for the decision in a system with an improper constellation, minimization of the modified cost function in (6) will result in a better estimator [11].

The modified MSE function can be written as follows

$$
\begin{aligned}
\eta_{n} & =\mathrm{E}\left[\left|e_{n}\right|^{2}\right]=0.25 \sigma_{s}^{2}\left(\mathbf{g}_{n}^{\mathcal{H}} \mathbf{H} \mathbf{V}_{n} \mathbf{H}^{\mathcal{H}} \mathbf{g}_{n}+\mathbf{g}_{n}^{\mathcal{H}} \mathbf{H} \mathbf{V}_{n} \mathbf{H}^{\mathcal{T}} \mathbf{g}_{n}^{*}\right. \\
& \left.+\mathbf{g}_{n}^{\mathcal{T}} \mathbf{H}^{*} \mathbf{V}_{n} \mathbf{H}^{\mathcal{H}} \mathbf{g}_{n}+\mathbf{g}_{n}^{\mathcal{T}} \mathbf{H}^{*} \mathbf{V}_{n} \mathbf{H}^{\mathcal{T}} \mathbf{g}_{n}^{*}\right) \\
& -0.5 \sigma_{s}^{2}\left(\mathbf{g}_{n}^{\mathcal{H}} \mathbf{h}_{n}+\mathbf{g}_{n}^{\mathcal{T}} \mathbf{h}_{n}^{*}+\mathbf{h}_{n}^{\mathcal{H}} \mathbf{g}_{n}+\mathbf{h}_{n}^{\mathcal{T}} \mathbf{g}_{n}^{*}\right) \\
& +\sigma_{v}^{2}\left(\mathbf{g}_{n}^{\mathcal{H}} \mathbf{g}_{n}+\mathbf{g}_{n}^{\mathcal{T}} \mathbf{g}_{n}^{*}\right)+\sigma_{s}^{2} .
\end{aligned}
$$

Setting the partial derivative of $\eta_{n}$ with respect to $\mathbf{g}_{n}$ to zero results in the following equation

$$
2 \mathbf{h}_{n}=\mathbf{H} \mathbf{V}_{n} \mathbf{H}^{\mathcal{H}} \mathbf{g}_{n}+\mathbf{H} \mathbf{V}_{n} \mathbf{H}^{\mathcal{T}} \mathbf{g}_{n}^{*}+\frac{\sigma_{v}^{2}}{\sigma_{s}^{2}} \mathbf{g}_{n}
$$

The above equation holds since

$$
\begin{aligned}
\frac{\partial \mathbf{g}_{n}^{\mathcal{H}} \mathbf{g}_{n}}{\partial \mathbf{g}_{n}}=\frac{\partial \mathbf{g}_{n}^{\mathcal{T}} \mathbf{g}_{n}^{*}}{\partial \mathbf{g}_{n}}=\mathbf{g}_{n}^{*} ; \frac{\partial \mathbf{g}_{n}^{\mathcal{T}} \mathbf{h}_{n}^{*}}{\partial \mathbf{g}_{n}}=\frac{\partial \mathbf{h}_{n}^{\mathcal{H}} \mathbf{g}_{n}}{\partial \mathbf{g}_{n}}=\mathbf{h}_{n}^{*} ; \\
\frac{\partial \mathbf{g}_{n}^{\mathcal{H}} \mathbf{h}_{n}}{\partial \mathbf{g}_{n}}=\frac{\partial \mathbf{h}_{n}^{\mathcal{T}} \mathbf{g}_{n}^{*}}{\partial \mathbf{g}_{n}}=\mathbf{0}, \\
\frac{\partial \mathbf{g}_{n}^{\mathcal{H}} \mathbf{H} \mathbf{V}_{n} \mathbf{H}^{\mathcal{H}} \mathbf{g}_{n}}{\partial \mathbf{g}_{n}}=\left(\mathbf{H} \mathbf{V}_{n} \mathbf{H}^{\mathcal{H}} \mathbf{g}_{n}\right)^{*} ; \\
\frac{\partial \mathbf{g}_{n}^{\mathcal{H}} \mathbf{H} \mathbf{V}_{n} \mathbf{H}^{\mathcal{T}} \mathbf{g}_{n}^{*}}{\partial \mathbf{g}_{n}}=\mathbf{0} ; \\
\frac{\partial \mathbf{g}_{n}^{\mathcal{T}} \mathbf{H}^{*} \mathbf{V}_{n} \mathbf{H}^{\mathcal{H}} \mathbf{g}_{n}}{\partial \mathbf{g}_{n}}=\left[\mathbf{H}^{*} \mathbf{V}_{n} \mathbf{H}^{\mathcal{H}}+\left(\mathbf{H}^{*} \mathbf{V}_{n} \mathbf{H}^{\mathcal{H}}\right)^{\mathcal{T}}\right] \mathbf{g}_{n} \\
=2 \mathbf{H}^{*} \mathbf{V}_{n} \mathbf{H}^{\mathcal{H}} \mathbf{g}_{n} ; \\
\frac{\partial \mathbf{g}_{n}^{\mathcal{T}} \mathbf{H}^{*} \mathbf{V}_{n} \mathbf{H}^{\mathcal{T}} \mathbf{g}_{n}^{*}}{\partial \mathbf{g}_{n}}=\mathbf{H}^{*} \mathbf{V}_{n} \mathbf{H}^{\mathcal{T}} \mathbf{g}_{n}^{*} ;
\end{aligned}
$$

\section{Denoting}

$$
\begin{array}{rlrl}
\mathbf{H V} & \mathbf{H}^{\mathcal{H}}=\mathbf{A}_{r}+j \mathbf{A}_{i} ; & \mathbf{g}_{n}=\mathbf{g}_{r}+j \mathbf{g}_{i} ; \\
\mathbf{H} \mathbf{V}_{n} \mathbf{H}^{\mathcal{T}}=\mathbf{B}_{r}+j \mathbf{B}_{i} ; & \mathbf{h}_{n}=\mathbf{h}_{r}+j \mathbf{h}_{i},
\end{array}
$$

then Equ. (7) can be reformed as

$2 \mathbf{h}_{r}+2 j \mathbf{h}_{i}=$

$\left(\mathbf{A}_{r}+j \mathbf{A}_{i}\right)\left(\mathbf{g}_{r}+j \mathbf{g}_{i}\right)+\left(\mathbf{B}_{r}+j \mathbf{B}_{i}\right)\left(\mathbf{g}_{r}-j \mathbf{g}_{i}\right)+\xi\left(\mathbf{g}_{r}+j \mathbf{g}_{i}\right)$

$=\left(\mathbf{A}_{r} \mathbf{g}_{r}-\mathbf{A}_{i} \mathbf{g}_{i}\right)+j\left(\mathbf{A}_{r} \mathbf{g}_{i}+\mathbf{A}_{i} \mathbf{g}_{r}\right)$

$+\left(\mathbf{B}_{r} \mathbf{g}_{r}+\mathbf{B}_{i} \mathbf{g}_{i}\right)+j\left(\mathbf{B}_{i} \mathbf{g}_{r}-\mathbf{B}_{r} \mathbf{g}_{i}\right)+\xi \mathbf{g}_{r}+j \xi \mathbf{g}_{i}$

$=\left(\mathbf{A}_{r} \mathbf{g}_{r}-\mathbf{A}_{i} \mathbf{g}_{i}+\mathbf{B}_{r} \mathbf{g}_{r}+\mathbf{B}_{i} \mathbf{g}_{i}+\xi \mathbf{g}_{r}\right)$

$+j\left(\mathbf{A}_{r} \mathbf{g}_{i}+\mathbf{A}_{i} \mathbf{g}_{r}+\mathbf{B}_{i} \mathbf{g}_{r}-\mathbf{B}_{r} \mathbf{g}_{i}+\xi \mathbf{g}_{i}\right)$,

where $\xi=\frac{\sigma_{v}^{2}}{\sigma_{s}^{2}}$. The real and imaginary parts of the vector $2 \mathbf{h}$ can be expressed separately as

$$
\begin{aligned}
2 \mathbf{h}_{r} & =\mathbf{A}_{r} \mathbf{g}_{r}-\mathbf{A}_{i} \mathbf{g}_{i}+\mathbf{B}_{r} \mathbf{g}_{r}+\mathbf{B}_{i} \mathbf{g}_{i}+\xi \mathbf{g}_{r} \\
& =\left(\mathbf{A}_{r}+\mathbf{B}_{r}+\xi\right) \mathbf{g}_{r}+\left(\mathbf{B}_{i}-\mathbf{A}_{i}\right) \mathbf{g}_{i} \\
2 \mathbf{h}_{i} & =\mathbf{A}_{r} \mathbf{g}_{i}+\mathbf{A}_{i} \mathbf{g}_{r}+\mathbf{B}_{i} \mathbf{g}_{r}-\mathbf{B}_{r} \mathbf{g}_{i}+\xi \mathbf{g}_{i} \\
& =\left(\mathbf{A}_{i}+\mathbf{B}_{i}\right) \mathbf{g}_{r}+\left(\mathbf{A}_{r}-\mathbf{B}_{r}+\xi\right) \mathbf{g}_{i},
\end{aligned}
$$

or in vector form as

$$
\left[\begin{array}{c}
2 \mathbf{h}_{r} \\
2 \mathbf{h}_{i}
\end{array}\right]=\left[\begin{array}{cc}
\mathbf{A}_{r}+\mathbf{B}_{r}+\xi & \mathbf{B}_{i}-\mathbf{A}_{i} \\
\mathbf{A}_{i}+\mathbf{B}_{i} & \mathbf{A}_{r}-\mathbf{B}_{r}+\xi
\end{array}\right]\left[\begin{array}{l}
\mathbf{g}_{r} \\
\mathbf{g}_{i}
\end{array}\right]
$$

leading to the improved MMSE filter $\mathbf{g}_{n}=\mathbf{g}_{r}+j \mathbf{g}_{i}$, where $\mathbf{g}_{r}$ and $\mathbf{g}_{i}$ are derived as

$$
\left[\begin{array}{l}
\mathbf{g}_{r} \\
\mathbf{g}_{i}
\end{array}\right]=\left[\begin{array}{cc}
\mathbf{A}_{r}+\mathbf{B}_{r}+\xi & \mathbf{B}_{i}-\mathbf{A}_{i} \\
\mathbf{A}_{i}+\mathbf{B}_{i} & \mathbf{A}_{r}-\mathbf{B}_{r}+\xi
\end{array}\right]^{-1}\left[\begin{array}{l}
2 \mathbf{h}_{r} \\
2 \mathbf{h}_{i}
\end{array}\right] .
$$

The ZF solution is derived by minimizing the following function with respect to $\mathrm{g}_{n}$

$$
\begin{aligned}
\eta_{n}^{\prime} & =0.25 \sigma_{s}^{2}\left(\mathbf{g}_{n}^{\mathcal{H}} \mathbf{H} \mathbf{V}_{n} \mathbf{H}^{\mathcal{H}} \mathbf{g}_{n}+\mathbf{g}_{n}^{\mathcal{H}} \mathbf{H} \mathbf{V}_{n} \mathbf{H}^{\mathcal{T}} \mathbf{g}_{n}^{*}\right. \\
& \left.+\mathbf{g}_{n}^{\mathcal{T}} \mathbf{H}^{*} \mathbf{V}_{n} \mathbf{H}^{\mathcal{H}} \mathbf{g}_{n}+\mathbf{g}_{n}^{\mathcal{T}} \mathbf{H}^{*} \mathbf{V}_{n} \mathbf{H}^{\mathcal{T}} \mathbf{g}_{n}^{*}\right)+\sigma_{s}^{2} \\
& -0.5 \sigma_{s}^{2}\left(\mathbf{g}_{n}^{\mathcal{H}} \mathbf{h}_{n}+\mathbf{g}_{n}^{\mathcal{T}} \mathbf{h}_{n}^{*}+\mathbf{h}_{n}^{\mathcal{H}} \mathbf{g}_{n}+\mathbf{h}_{n}^{\mathcal{T}} \mathbf{g}_{n}^{*}\right) .
\end{aligned}
$$

Following the same procedure as shown previously, we can form the improved ZF filter as $\mathbf{g}_{n}^{\prime}=\mathbf{g}_{r}^{\prime}+j \mathbf{g}_{i}^{\prime}$, where $\mathbf{g}_{r}^{\prime}$ and $\mathbf{g}_{i}^{\prime}$ are derived as

$$
\left[\begin{array}{l}
\mathbf{g}_{r}^{\prime} \\
\mathbf{g}_{i}^{\prime}
\end{array}\right]=\left[\begin{array}{cc}
\mathbf{A}_{r}+\mathbf{B}_{r} & \mathbf{B}_{i}-\mathbf{A}_{i} \\
\mathbf{A}_{i}+\mathbf{B}_{i} & \mathbf{A}_{r}-\mathbf{B}_{r}
\end{array}\right]^{-1}\left[\begin{array}{c}
2 \mathbf{h}_{r} \\
2 \mathbf{h}_{i}
\end{array}\right]
$$

Unlike the MMSE solution, the ZF filter does not need any knowledge of the noise as shown by (10).

The computation of the improved filters $\mathbf{g}_{n}$ and $\mathbf{g}_{n}^{\prime}$ is slightly more complex than the computation of the conventional filters $\mathbf{w}_{n}$ and $\mathbf{w}_{n}^{\prime}$ in (4) due to the inversion of a dimension doubled matrix. However, once the filter coefficients are derived, the proposed and the conventional systems have exactly the same processing complexity in the receiver. Therefore, the complexity increase by the improved schemes is not significant compared to the conventional schemes, especially in slow-fading channels for which the filter coefficients do not need to be updated frequently.

In what follows, we use QPSK/OQPSK and 4-ASK systems as example to demonstrate how the vector $\overline{\mathbf{s}}_{n}$ in (3) and the 
matrix $\mathbf{V}_{n}$ in (5) can be derived in order to carry out the iterative process. The filter output can be expressed as

$$
z_{n}=\mathbf{g}_{n}^{\mathcal{H}} \mathbf{r}_{n}=\mu_{n} s_{n}+\nu_{n},
$$

where the combined noise and residual interference $\nu_{n}$ can be approximated as a Gaussian random variable [12], i.e., $\nu_{n} \sim$ $\mathcal{C N}\left(0, N_{\nu}\right)$. The parameters $\mu_{n}, N_{\nu}$ can be determined as [13]

$$
\begin{aligned}
& \mu_{n}=\mathrm{E}\left\{z_{n} s_{n}^{*}\right\}=\mathbf{g}_{n}^{\mathcal{H}} \mathrm{E}\left[\mathbf{r}_{n} s_{n}^{*}\right]=\mathbf{g}_{n}^{\mathcal{H}} \mathbf{C}_{\mathbf{r} s} ; \\
& N_{\eta}=\mu_{n}-\mu_{n}^{2},
\end{aligned}
$$

where $\mathbf{C}_{\mathbf{r} s}=\mathrm{E}\left[\mathbf{r}_{n} s_{n}^{*}\right]=\mathbf{h}_{n}$. After computing the values of $\mu_{n}$ and $N_{\nu}$, the conditional probability density function (PDF) of the filter output can be obtained as

$$
f\left(z_{n} \mid s_{n}=s_{m}\right)=\frac{1}{\pi N_{\nu}} \exp \left(-\frac{\left|z_{n}-\mu_{n} s_{m}\right|^{2}}{N_{\nu}}\right),
$$

For QPSK/OQPSK and 4-ASK systems, each symbol $s_{n}$ corresponds to two information bits, denoted as $b_{n}^{0}$ and $b_{n}^{1}$. For the 4-ASK system, the log-likelihood ratio (LLR) for the first information bit $b_{n}^{0}$ can be computed as

$$
\begin{aligned}
\lambda\left(b_{n}^{0}\right) & =\ln \frac{f\left(z_{n} \mid b_{n}^{0}=0\right)}{f\left(z_{n} \mid b_{n}^{0}=1\right)}=\ln \frac{f\left(z_{n} \mid s_{n}=s_{0}\right)+f\left(z_{n} \mid s_{n}=s_{3}\right)}{f\left(z_{n} \mid s_{n}=s_{1}\right)+f\left(z_{n} \mid s_{n}=s_{2}\right)} \\
& \approx \ln \frac{\exp \left(-\left|z_{n}-\mu_{n} s_{n}^{+}\right|^{2} / N_{\nu}\right)}{\exp \left(-\left|z_{n}-\mu_{n} s_{n}^{-}\right|^{2} / N_{\nu}\right)} \\
& =\frac{1}{N_{\nu}}\left\{\left|z_{n}-\mu_{n} s_{n}^{-}\right|^{2}-\left|z_{n}-\mu_{n} s_{n}^{+}\right|^{2}\right\} \\
& =\frac{1}{1-\mu_{n}} \operatorname{Re}\left\{\left[2 s_{n}^{+*} z_{n}-\mu_{n}\left|s_{n}^{+}\right|^{2}\right]-\left[2 s_{n}^{-*} z_{n}-\mu_{n}\left|s_{n}^{-}\right|^{2}\right]\right\},
\end{aligned}
$$

where $s_{+}$denotes the 4-ASK symbol corresponding to $\max \left\{f\left(z_{n} \mid s_{0}\right), f\left(z_{n} \mid s_{3}\right)\right\}$, and $s_{-}$denotes the 4-ASK symbol corresponding to $\max \left\{f\left(z_{n} \mid s_{1}\right), f\left(z_{n} \mid s_{2}\right)\right\}$ since the real part of the symbols $s_{0}, s_{3}$ corresponds to 0 , and the real part of the symbols $s_{1}, s_{2}$ corresponds to 1 as shown in Fig. 1.

For the QPSK/OQPSK system, since all the signal candidates have the same energy, i.e., $\left|s_{0}\right|^{2}=\left|s_{1}\right|^{2}=\left|s_{2}\right|^{2}=\left|s_{3}\right|^{2}$, the LLR value of $b_{n}^{0}$ can thus be simplified to

$$
\lambda\left(b_{n}^{0}\right) \approx \frac{2}{1-\mu_{n}} \operatorname{Re}\left\{s_{n}^{+*} z_{n}-s_{n}^{-*} z_{n}\right\},
$$

and the definition of $s_{+}$and $s_{-}$is the same as described previously. The LLR value for the second information bit can be obtained in a similar manner as

$$
\begin{aligned}
& \lambda\left(b_{n}^{1}\right)=\ln \frac{f\left(z_{n} \mid s_{0}\right)+f\left(z_{n} \mid s_{1}\right)}{f\left(z_{n} \mid s_{2}\right)+f\left(z_{n} \mid s_{3}\right)} \\
& \approx \begin{cases}\frac{1}{1-\mu_{n}} \operatorname{Re}\left\{\left[2 s_{n}^{+*} z_{n}-\mu_{n}\left|s_{n}^{+}\right|^{2}\right]-\left[2 s_{n}^{-*} z_{n}-\mu_{n}\left|s_{n}^{-}\right|^{2}\right]\right\} ; \\
\frac{2}{1-\mu_{n}} \operatorname{Re}\left\{s_{n}^{+*} z_{n}-s_{n}^{-*} z_{n}\right\} ; & \text { for } 4 \text {-ASK QPSK/OQPSK }\end{cases}
\end{aligned}
$$

where $s_{+}$denotes the 4-ASK/QPSK/OQPSK symbol corresponding to $\max \left\{f\left(z_{n} \mid s_{0}\right), f\left(z_{n} \mid s_{1}\right)\right\}$, and $s_{-}$ denotes the 4-ASK/QPSK/OQPSK symbol corresponding to $\max \left\{f\left(z_{n} \mid s_{2}\right), f\left(z_{n} \mid s_{3}\right)\right\}$ since the imaginary part of the symbols $s_{0}, s_{1}$ corresponds to 0 , and the imaginary part of the symbols $s_{2}, s_{3}$ corresponds to 1 as shown in Figs. 1 and 2.
Fig. 1. 4-ASK constellation and bit-to-symbol mapping.

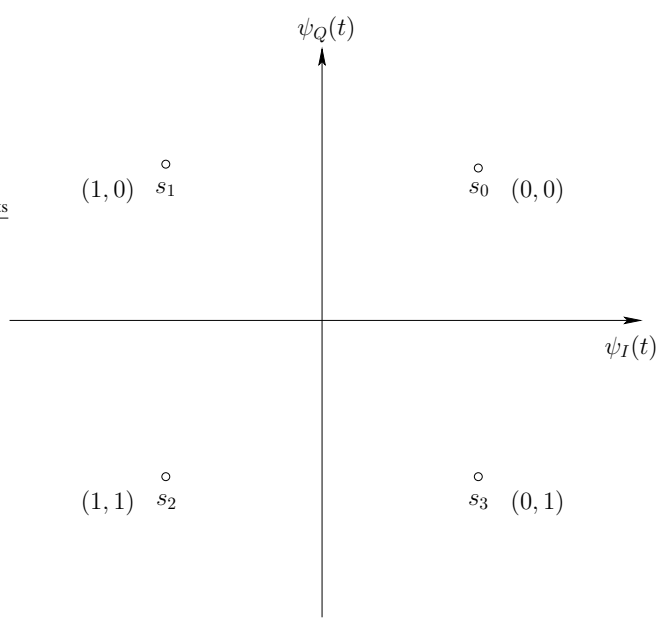

Fig. 2. QPSK/OQPSK constellation and bit-to-symbol mapping.

Since the a priori probability of each symbol $P_{r}\left(s_{j}\right)=$ $P_{r}\left(b_{j}^{0}\right) \cdot P_{r}\left(b_{j}^{1}\right)$ in an uncoded system, according to the symbolto-bit mapping shown in Figs. 1 and 2, we have

$$
\begin{aligned}
& P_{r}\left(s_{j}=s_{0}\right)=P_{r}\left(b_{j}^{0}=0\right) \cdot P_{r}\left(b_{j}^{1}=0\right) \\
& P_{r}\left(s_{j}=s_{1}\right)=P_{r}\left(b_{j}^{0}=1\right) \cdot P_{r}\left(b_{j}^{1}=0\right) \\
& P_{r}\left(s_{j}=s_{2}\right)=P_{r}\left(b_{j}^{0}=1\right) \cdot P_{r}\left(b_{j}^{1}=1\right) \\
& P_{r}\left(s_{j}=s_{3}\right)=P_{r}\left(b_{j}^{0}=0\right) \cdot P_{r}\left(b_{j}^{1}=1\right)
\end{aligned}
$$

where

$$
\begin{array}{ll}
P_{r}\left(b_{j}^{0}=0\right)=\frac{e^{\lambda\left(b_{j}^{0}\right)}}{1+e^{\lambda\left(b_{j}^{0}\right)}} ; & P_{r}\left(b_{j}^{0}=1\right)=\frac{1}{1+e^{\lambda\left(b_{j}^{0}\right)}} \\
P_{r}\left(b_{j}^{1}=0\right)=\frac{e^{\lambda\left(b_{j}^{1}\right)}}{1+e^{\lambda\left(b_{j}^{1}\right)}} ; & P_{r}\left(b_{j}^{1}=1\right)=\frac{1}{1+e^{\lambda\left(b_{j}^{1}\right)}}
\end{array}
$$

With the a priori probability of each symbol $P_{r}\left(s_{j}\right)$, the soft estimate $\bar{s}_{j}$ in the vector $\overline{\mathbf{s}}_{n}$ and the variance $\operatorname{var}\left(s_{j}\right)$ in the matrix $\mathbf{V}_{n}$, respectively, can be calculated as [13]

$$
\begin{aligned}
& \bar{s}_{j}=\mathrm{E}\left\{s_{j}\right\}=\sum_{m=0}^{3} s_{m} P_{r}\left(s_{j}=s_{m}\right) ; \\
& \operatorname{var}\left(s_{j}\right)=\mathrm{E}\left[\left|s_{j}\right|^{2}\right]-\left|\bar{s}_{j}\right|^{2},
\end{aligned}
$$

where $\mathrm{E}\left[\left|s_{j}\right|^{2}\right]=\sum_{m=0}^{3}\left|s_{m}\right|^{2} P_{r}\left(s_{j}=s_{m}\right)$.

\section{NumericAl RESUlts}

We compare different iterative algorithms by applying them to uncoded MIMO systems. The transmit power is set to $N_{t} \sigma_{s}^{2}=N_{t}$, i.e., unit average transmit power is used for each transmitted symbol. We assume uncorrelated Rayleigh fading channel and the channel matrix is normalized such that $\mathrm{E}\left[\|\mathbf{H}\|_{F}^{2}\right]=1$. In Figs. $3-5$, the number of antennas are set to be $N_{t}=N_{r}=4$; while in Figs. 6, $N_{r}$ takes 
Iterative $\mathrm{ZF}$ receiver, $\mathrm{N}_{\mathrm{t}}=4, \mathrm{~N}_{\mathrm{r}}=4$

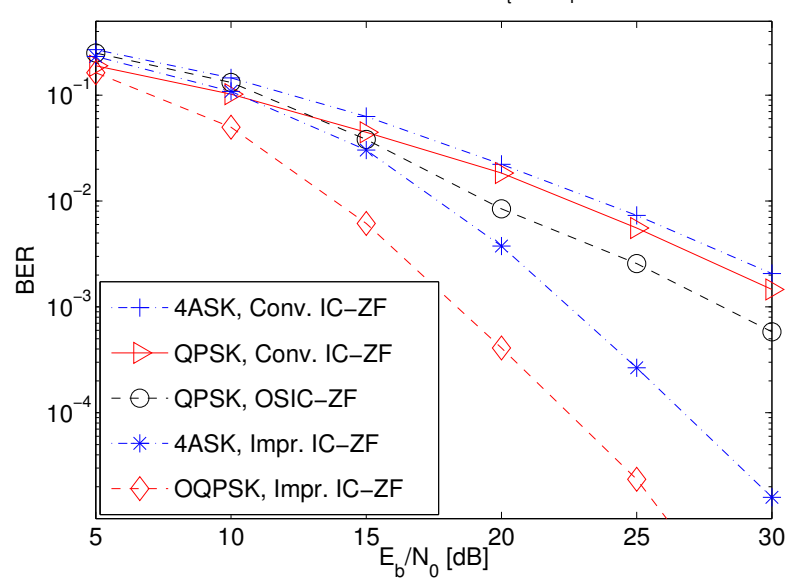

Fig. 3. Performance comparison of different iterative IC-ZF receivers for 4-ASK, QPSK, OQPSK. The curves corresponding to the iterative schemes are plotted at the 3rd iteration when the systems reach convergence.

the values $2,3,4,5$, and the number of transmit antennas is fixed to $N_{t}=4$. The employed modulation schemes are 4ASK, QPSK, OQPSK, which are chosen such that all the systems have the same data transmission rate or spectrum efficiency. The simulation results are averaged over at least 50,000 channel realizations.

Figs. 3 and 4 show the performance comparison of iterative $\mathrm{ZF}$ and MMSE schemes for different systems. Direct implementation of the ZF scheme expressed by (10) leads to the numerical problem caused by inversion of an ill-conditioned matrix. However, the problem can be easily resolved by Tikhonov regularization, and the regularization parameter is chosen to be a very small number. The results are shown at the 3rd iteration since we have observed that it takes no more than 3 iterations for the iterative algorithms to reach steady state, and further iterations do not yield noticeable performance improvement. One can see from the figures that with the conventional IC-ZF/MMSE algorithms, the QPSK modulated system outperforms the 4-ASK system. The OQPSK system with the improved schemes achieve significant performance gains compared to all the other systems. The curves for the OQPSK system with conventional schemes are omitted in the figures since they are identical to that of the QPSK system when conventional schemes are applied. By comparison, the 4-ASK system with the improved IC-ZF scheme performs much better than the QPSK system with the conventional IC$\mathrm{ZF}$ scheme. However, in the MMSE case, the QPSK system has better performance at low SNRs, while it is outperformed by the 4-ASK system with the improved IC-MMSE scheme at high SNR.

In Figs. 3 and 4, we also show the performance of the ordered successive interference cancellation (OSIC) algorithm [14], [15], and compare it with other schemes. The principle of the OSIC is to decode the "strongest" signal first, and cancel its contribution from the received signal, then proceed to decode the "strongest" of the remaining signal, and so on. The detection scheme is also referred to

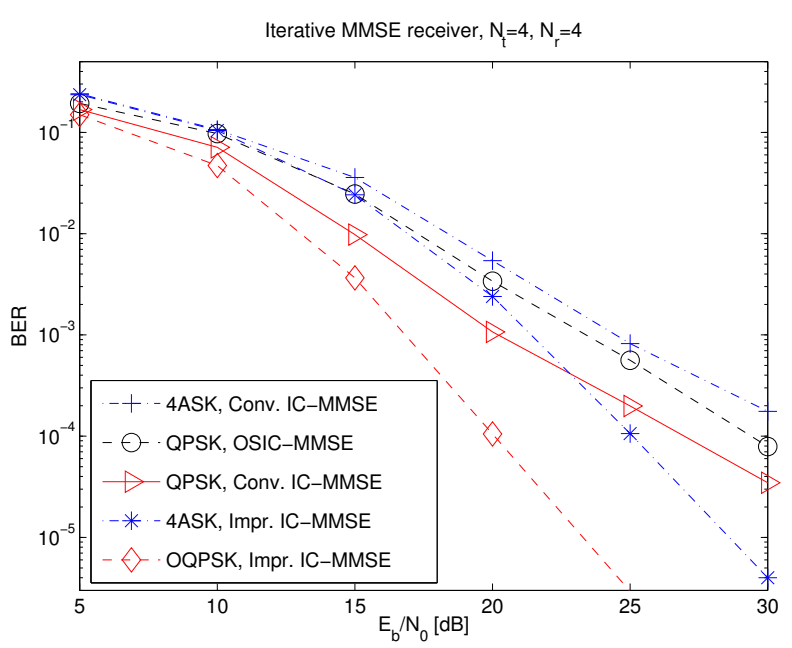

Fig. 4. Performance comparison of different iterative IC-MMSE receivers for 4-ASK, QPSK, OQPSK. The curves corresponding to the iterative schemes are plotted at the 3rd iteration when the systems reach convergence.

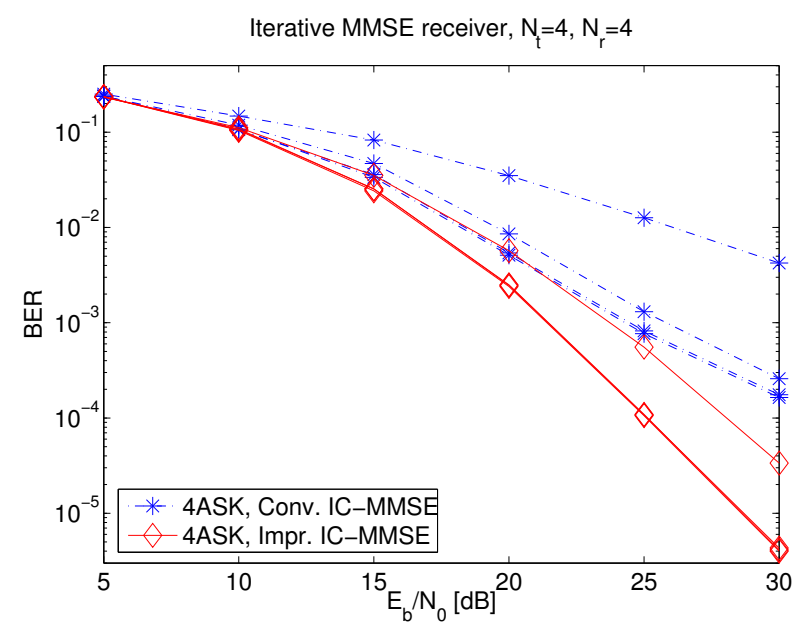

Fig. 5. Performance comparison of the iterative IC-MMSE schemes for the 4-ASK system.

as sequential nulling and cancellation. The optimal detection order is from the strongest to the weakest signal in order to minimize the error propagation from one detection step to the next. The nulling operation can be performed by means of ZF (OSIC-ZF) or MMSE (OSIC-MMSE). One can see from the figures that under the ZF criterion, the QPSK system with OSIC yields better performance than the conventional QPSK and 4-ASK systems, however, it is outperformed by the conventional QPSK system in the MMSE case, meaning that successive interference cancellation is more vulnerable to the error propagation problem (even with the optimal detection order) than parallel interference cancellation with soft symbol estimates. In all the cases, the OQPSK and 4-ASK systems with the proposed iterative schemes yield much improved performance than the QPSK system with OSIC.

In Fig. 5, we examine the performance of the iterative ICMMSE schemes for the 4-ASK system at different iterations. The topmost curve represents the initial MIMO detection 
Iterative MMSE receiver for 4ASK

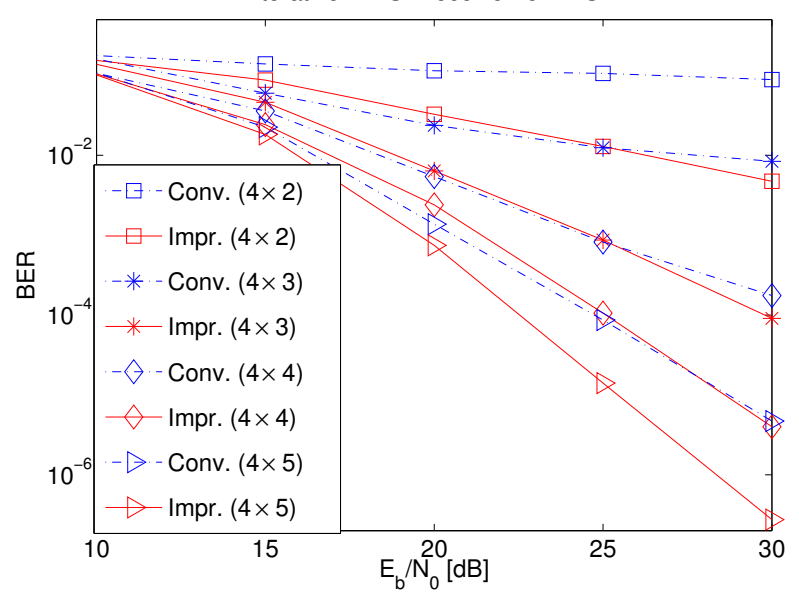

Fig. 6. Performance comparison of the iterative IC-MMSE schemes for the 4-ASK system with different antenna setups. All the curves are plotted at the 3rd iteration when the systems reach convergence.

without interference cancellation. In this case, the soft symbol estimate vector $\overline{\mathbf{s}}_{n}$ in (3) is initialized to zero. At subsequent stages, the system switches to decision directed mode and uses the soft symbol estimate from the previous iteration to remove the effect of CAI. In addition to the superior performance achieved by the improved scheme, we also notice that the conventional system has to iterate 3 times to converge, while only 2 iterations are needed for the system to reach convergence with the improved IC-MMSE scheme. Therefore, the proposed algorithm also leads to faster convergence for the iterative MIMO receiver.

Fig. 6 shows the performance of the iterative IC-MMSE schemes for the 4-ASK system with antenna configurations of 4 transmit antennas and 2 to 5 receive antennas. In all the cases, the proposed scheme shows clear superiority over the conventional scheme, however, the performance gain becomes smaller as the number of receive antennas increases, which indicates that it is more advantageous to apply the proposed scheme with fewer receive antennas than transmit antennas. Interestingly, the performance of the proposed scheme in $4 \times 2$, $4 \times 3,4 \times 4$ systems is comparable to that of the conventional scheme in $4 \times 3,4 \times 4,4 \times 5$ systems, respectively. This is equivalent to saying that the proposed scheme has the effect of increasing receive diversity order.

\section{CONCLUSiOnS}

In this paper, we propose a novel iterative receiver for MIMO systems, which is derived by optimization of modified cost functions for improper constellations. The numerical results reveal that the proposed schemes for the systems with improper constellations achieve superior performance and faster convergence than the conventional systems with both improper and proper constellations, and the performance advantages are more obvious in the situation when the number of receive antennas is less than the number of transmit antennas. The proposed iterative receiver strategy provides a reliable and practical solution to high data-rate transmission for wireless communications.

\section{ACKNOWLEDGEMENT}

The financial support of the UK Engineering and Physical Sciences Research Council (under grant number EP/D07827X/1) is gratefully acknowledged.

\section{REFERENCES}

[1] G. Foschini. "Layered space-time architecture for wireless communication in a fading environment when using multiple-element antennas", Bells Labs Tech. J., vol. 1, pp. 41-59, 1996.

[2] G. Foschini, M. Gans. "On limits of wireless communications in a fading environment when using multiple antennas", Wireless Personal Commun., vol. 6, no. 3, pp. 311-335, March 1998.

[3] A. Paulraj, R. Nabar, D. Gore. Introduction to Space-Time Wireless Communications, Cambridge, University Press, 2003.

[4] M. Sellathurai, S. Haykin. "Turbo-BLAST for wireless communications: theory and experiments", IEEE Trans. Sig. Proc., vo. 50, no. 10, pp. 2538-2546, Oct. 2002.

[5] A. Tonello. "Space-time bit-interleaved coded modulation with an iterative decoding strategy", Proc. VTC Fall'00, pp. 473-478, Sept. 2000.

[6] C. Douillard, M. Jezequel, C. Berrou. "Iterative correction of intersymbol interference: turbo-equalization”. European Trans. on Telecommun., pp. 507-511, Sept. 1995.

[7] X. Wautelet, A. Dejonghe, L. Vandendorpe. "MMSE-based fractional turbo receiver for space-time BICM over frequency-selective MIMO fading channels", IEEE Trans. Sig. Proc., vo. 52, no. 6, pp. 1804-1809, June 2004.

[8] J. Wang, S. Li. "Reliability based reduced-complexity MMSE soft interference cancellation MIMO turbo receiver", Proc. PIMRC, pp. 1-4, Sept. 2007.

[9] X. Wang, H. Poor. "Iterative (turbo) soft interference cancellation and decoding for coded CDMA". IEEE Transactions on Communications, vol. 47, pp. 1046-1061, July 1999.

[10] M. Tuchler, R. Koetter, A. Singer. "Turbo equalization: principles and new results", IEEE Trans. on Commun., vol. 50, pp. 754-767, May 2002.

[11] S. Buzzi, M. Lops, A. Tulino. "A new family of MMSE multiuser receivers for interference suppression in DS/CDMA systems employing BPSK modulation". IEEE Transactions on Communications, vol. 49, no. 1, pp. 154-167, Jan. 2001.

[12] V. Poor, S. Verdu. "Probability of error in MMSE multiuser detection", IEEE Trans. on Commun., vol. 43, pp. 858-971, May 1997.

[13] A. Dejonghe and L. Vandendorpe. "Turbo-equalization for multilevel modulation: an efficient low-complexity scheme", IEEE International Conference on Communications, vol. 3, pp. 1863 - 1867, 2002.

[14] P. Wolniansky, G. Foschini, G. Golden, R. Valenzuela. "V-BLAST: an architecture for realizing very high data rates over the rich-scattering wireless channel", URSI International Symposium on Signals, Systems, and Electronics (ISSSE), pp. 295-300, 1998.

[15] G. Golden, G. Foschini, R. Valenzuela, P. Wolniansky. "Detection algorithm and initial laboratory results using the V-BLAST space-time communication architecture", IEE Electronic Letters, vol. 35, no. 1, pp. 14-16, January 1999. 